\title{
Preemptive Nebulization of Lidocaine Epinephrine Before Anesthesia for Rigid Bronchoscopy in Pediatric: A Randomized Controlled Study
}

Hosam I. El Said Saber,* T. El-Desoky, $\dagger$ Mostafa S. Elawady,* Hani I. Taman*

$*$ From Anaesthesia and Surgical Intensive Care Department, $\uparrow$ From Pediatrics Department, Faculty of Medicine, Mansoura University, Egypt

Corresponding author: Hani I. Taman, Mobile: +201008288242, E-mail: hani_taman@hotmail.com

\begin{abstract}
Background: Foreign body aspiration in pediatrics is usually managed by rigid bronchoscopy, which is associated with plenty of adverse events. Objective: We tried to compare the effect of nebulized saline, lidocaine or combined lidocaine with epinephrine on postoperative respiratory complications.

Patients and methods: This prospective study included 90 children, who were divided into three groups according to the nebulized solution; NS group (normal saline 0.9\%), L group (lidocaine $1 \% 4 \mathrm{mg} \cdot \mathrm{kg}^{-1}$ ) and LA group [4 mg. $\mathrm{kg}^{-1}$ lidocaine $1 \%$ and adrenaline $(1: 1000) 3 \mathrm{mg}$ ). Our primary outcome was the incidence of post-operative respiratory complications, while the secondary ones included hemodynamic changes and the incidence of intraoperative cough or desaturation. Results: All pre-procedural data were insignificant among the three groups. The LA group expressed higher heart rates, while the L group showed a significant reduction when compared to NS group. Propofol consumption showed a significant decline in two studied groups compared to the NS group. Intraoperative cough was higher in NS group in comparison to L and LA groups. Although, intraoperative desaturation per case along with post-operative sedation showed no significant difference among the three groups, post-operative cough frequency attacks and severity were higher in NS group when compared to L and LA group and when L group were compared to LA group. Postoperative stridor was insignificant among the three groups.

Conclusion: Nebulized lidocaine/adrenaline combination is appropriate option to achieve proper intraoperative sedation and upper airway conditions with reduction of post-operative negative respiratory outcomes together with minor hemodynamic changes.
\end{abstract}

Keywords: Rigid bronchoscopy; Nebulization; Lidocaine; Epinephrine.

\section{INTRODUCTION}

Foreign body aspiration by children is a common problem that is frequently encountered in emergency pediatric practice. Impaction of the inhaled foreign body could lead to asphyxia, and it is the major cause of mortality in children aged less than four years ${ }^{1}$. Therefore, this serious entity should be immediately managed to avoid subsequent complications and irreversible lung damage ${ }^{2}$. It was managed by emetics and purges in the $19^{\text {th }}$ century, but these management modalities were ineffective and were associated with a $23 \%$ mortality rate ${ }^{3}$. However, this rate dramatically decreased following the intervention of bronchoscopy ${ }^{4}$.

For more than a century, rigid bronchoscopy has been a crucial tool in the diagnosis and treatment of various airway pathologies ${ }^{5}$. Despite the widespread use of flexible bronchoscopy, the rigid one is still used as the gold standard option for many complex airway diseases, including foreign body aspiration ${ }^{6,7}$. It allows the operator to properly visualize and manipulate the aspirated object, with proper management of mucosal haemorrhage, if it occurred ${ }^{8}$. Although rigid bronchoscopy has a $95 \%$ success rate with a safe profile (less than $1 \%$ complication rate) 9, 10, multiple complications could be encountered, including bronchospasm, laryngeal oedema, pneumothorax, tracheobronchial lacerations, hypoxic brain damage, infection, bleeding and cardiac arrest 1,11, 12. Although early clinicians used topical anaesthesia for rigid bronchoscopy interventions ${ }^{1}$, most anaesthetists prefer general anaesthesia nowadays, thanks to advances in anaesthetic delivery and the increased experience with rigid bronchoscopy procedures $7,{ }^{13}$. During the procedure, anaesthetists usually try to maintain an adequate depth of anaesthesia to prevent cough and straining during the intervention. Stable hemodynamics and rapid post-procedural recovery are crucial needs as well ${ }^{14,15}$. Lidocaine is an inexpensive, widely available anaesthetic agent with an excellent safety profile when nebulized. This contrasts the fact that topical anaesthetics are rapidly absorbed into the circulation following application onto mucosal surfaces, which increases the risk of systemic adverse events ${ }^{15}$. Epinephrine (adrenaline) is a potent adrenergic alpha and beta receptor stimulant. It is used during cardiopulmonary resuscitation. In addition, it could be nebulized in pediatric patients with airway inflammatory diseases like croup and bronchiolitis ${ }^{16,17}$. Its potential positive impact on the airway is due to a decrease in airway secretions and oedema (alpha effect), together with airway dilatation and inhibition of inflammatory cascades (beta effect) ${ }^{18}$.

Based on local anaesthetic effects of lidocaine and anti-edematous effects of adrenaline, our hypothesis is that preoperative nebulization of both 
drugs may improve outcomes after rigid bronchoscopy in the pediatric population.

Our primary outcome was to compare the incidence of post-operative respiratory complications (cough, stridor or desaturation) among the three studied groups. Secondary outcomes included hemodynamic changes and the incidence of intra-operative cough or desaturation (defined as oxygen saturation $<90 \%$ ). Any change detected in MAP or heart rate more than $20 \%$ of its basal values were considered significant events.

\section{PATIENTS AND METHODS}

This prospective randomized trial was conducted at Mansoura University Anesthesiology Department in collaboration with the Pediatric Department. The study was conducted over a period of two years, from April 2017 till April 2019.

\section{Sample size}

Priori $G$ power was done to calculate sample size, power of $80 \%$ was used with $\alpha$ error of 0.05 and effective size $35 \%$, the total number of patients obtained was 85 , a dropout of $5 \%$ was expected. Therefore, the total sample size was 90 patients. We included pediatric cases suspected of foreign body aspiration and scheduled for elective or emergency rigid bronchoscopy. Contrarily, we excluded children with known cardiovascular disorders or any congenital skeletal deformities.

\section{Ethical considerations:}

The study was approved by Institutional Review Board (IRB-MFM) of Mansoura University, Faculty of Medicine with Code number R/16.12.32, March 2017. We obtained informed written consent from the guardians of each participant after simple explanation of the indication, benefits, and possible complications of each intervention. This work has been carried out in accordance with The Code of Ethics of the World Medical Association (Declaration of Helsinki) for studies involving humans.

Using the sealed envelope method, the included 90 cases were divided into three equal groups according to the commenced preoperative nebulizer; NS group included 30 patients who received the nebulized solution containing only $0.9 \%$ saline, $\mathrm{L}$ group included another 30 cases who received nebulized solution containing $4 \mathrm{mg} \cdot \mathrm{kg}^{-1}$ lidocaine $1 \%$, and LA group included the remaining 30 cases who received nebulized solution containing $4 \mathrm{mg} \cdot \mathrm{kg}^{-1}$ lidocaine $1 \%$ in addition to $3 \mathrm{mg}$ of adrenaline $(1: 1000)$.

All patients were subjected to history taking, clinical examination and routine preprocedural investigations if needed. After planning the bronchoscope procedure, all patients were medicated with the nebulizer (according to the group allocation) about 15 minutes prior to the intervention. Then, all patients were monitored with AAGBI recommended basic monitoring on arrival to the operative theatre. Anaesthesia was induced by intravenous propofol (1.5 mg. $\left.\mathrm{kg}^{-}{ }^{1}\right)$, dexmedetomidine $\left(0.5 \mu \mathrm{g} . \mathrm{kg}^{-}{ }^{1}\right)$ and suxamethonium $\left(1 \mathrm{mg} . \mathrm{kg}^{-1}\right)$ to facilitate the rigid bronchoscope passage through the vocal cords. Anesthesia was maintained by propofol infusion $\left(150 \mu \mathrm{g} . \mathrm{kg}^{-1} \cdot \mathrm{min}^{-1}\right)$ and dexmedetomidine $(0.4 \mu \mathrm{g} . \mathrm{kg}$ ${ }^{1}$. $\left.\mathrm{min}^{-1}\right)$. An additional dose of propofol $0.5 \mathrm{mg} / \mathrm{kg}$ was given to alleviate intraoperative coughing. The bronchoscopy procedure was then performed by the same experienced bronchoscopist. The heart rate, mean arterial pressure and $\mathrm{SPO}_{2}$ were recorded at nebulizer intake, induction, bronchoscopy insertion and then at one, three, five, seven, and ten minutes during the procedure. Also, the number of cases who developed intra-operative cough, number of cough and desaturation attacks per every case were noted and recorded.

After the procedure, all patients were transferred to PACU and then to the internal ward, where all patients received humified oxygen for one hour. During the post-operative period, both MAP and heart rate were recorded at arrival, 15, 30 and 60 minutes after the procedure. Post-operative sedation was recorded according to the Richmond AgitationSedation Scale (RASS) ${ }^{19}$. Additionally, the incidence of post-operative stridor and cough was recorded. The severity of post-operative cough was graded using the VAS (a vertically marked scale of 1 to 10 , with mild cough $(<4)$, moderate $(4-6)$ and sever $>6)$.

\section{Statistical analysis}

IBM's SPSS statistics for MacOs (version 26) was used for the analysis of the collected and tabulated data. The Shapiro-Wilk test was done to ensure that the data distribution was normal. Continuous data with a regularly distributed distribution were expressed as mean $\pm \mathrm{SD}$, whereas categorical variables and those with an erratically distributed distribution were expressed as median and interquartile range or number and percentage (as appropriate). For normally and abnormally distributed continuous data, one-way ANOVA and Kruskal Wallis tests were utilized. The crosstabs function was used to run the Chi-square test on categorical data. All tests were carried out using a $95 \%$ confidence interval. A p (probability) value $\leq 0.05$ was considered statistically significant.

\section{RESULTS}

Starting with demographic data of the included children, their ages had mean values of 35.9, 37.93, and 34.8 months in the NS, L, and LA groups, respectively. Regarding gender distribution, boys represented $60 \%$, $60 \%$ and $70 \%$ of the included cases in the same three groups, respectively, while the remaining cases were girls. As regards the site of impacted foreign body, it was located in the trachea in $43.3 \%, 66.75 \%$, and $76.7 \%$ of cases in the same three groups, while the right bronchus was the site of impaction in 30\%, 20\% and $10 \%$ of cases in the same groups respectively. The left bronchus was affected in the remaining cases. All of the previous parameters showed no significant differences among the three groups ( $\mathrm{p}>0.05)$, as shown in table (1). 
Table (1): Demographic characteristics and foreign body site in the three groups

\begin{tabular}{|c|c|c|c|c|c|c|c|c|}
\hline & & $\begin{array}{l}\text { NS group } \\
(n=30)\end{array}$ & $\begin{array}{l}\text { L group } \\
(\mathbf{n}=\mathbf{3 0})\end{array}$ & $\begin{array}{l}\text { LA group } \\
\quad(n=30)\end{array}$ & $\mathbf{P}$ & $\mathbf{P 1}$ & $\mathbf{P 2}$ & P3 \\
\hline Age (r & nths) & $35.90 \pm 3.325$ & $37.93 \pm 5.192$ & $34.80 \pm 6.661$ & 0.069 & 0.410 & 1 & 0.069 \\
\hline Gender & Male & $18(60.0 \%)$ & $18(60.0 \%)$ & $21(70.0 \%)$ & & & & \\
\hline $\begin{array}{c}\text { (number \& } \\
\%)\end{array}$ & Female & $12(40.0 \%)$ & $12(40.0 \%)$ & $9(30.0 \%)$ & 0.650 & $>0.05$ & $>0.05$ & $>0.05$ \\
\hline Weig & (kg) & $16.03 \pm 2.51$ & $16.25 \pm 1.83$ & $17.04 \pm 2.99$ & 0.708 & 0.163 & 0.220 & 0.699 \\
\hline & Trachea & $13(43.3 \%)$ & $20(66.7 \%)$ & $23(76.7 \%)$ & & & & \\
\hline $\begin{array}{c}\text { Site } \\
\text { (number \& }\end{array}$ & $\begin{array}{c}\text { Right } \\
\text { bronchus }\end{array}$ & $9(30.0 \%)$ & $6(20.0 \%)$ & $3(10.0 \%)$ & 0.098 & $>0.05$ & $>0.05$ & $>0.05$ \\
\hline$\%)$ & $\begin{array}{c}\text { Left } \\
\text { bronchus }\end{array}$ & $8(26.7 \%)$ & $4(13.3 \%)$ & $4(13.3 \%)$ & & & & \\
\hline
\end{tabular}

P1: NS group vs L group. P2: NS group vs LA group. P3: L group vs LA group.

As shown in table (2), no significant difference was noted between the three study groups regarding their basal heart rates. However, on nebulizer administration, the LA group expressed significantly higher heart rates, while the L group showed a significant decrease when compared to NS group, and this was noticed throughout the intraoperative and postoperative periods.

Table (2): Intra- and post-operative follow-up of heart rate in the three groups.

\begin{tabular}{|c|c|c|c|c|c|c|c|c|}
\hline \multicolumn{2}{|c|}{ Heart rate (bpm) } & $\begin{array}{l}\text { NS group } \\
(n=30)\end{array}$ & $\begin{array}{l}\text { L group } \\
(n=30)\end{array}$ & $\begin{array}{l}\text { LA group } \\
(n=30)\end{array}$ & $\mathbf{P}$ & P1 & P2 & P3 \\
\hline \multirow{9}{*}{ 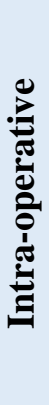 } & Basal & $142.60 \pm 11.106$ & $136.67 \pm 7.941$ & $140.90 \pm 10.203$ & 0.061 & 0.065 & 1 & 0.298 \\
\hline & Nebulizer & $142.67 \pm 11.469$ & $137.13 \pm 7.969$ & $152.27 \pm 12.537$ & $<0.001$ & 0.153 & 0.003 & $<0.001$ \\
\hline & Induction & $140.87 \pm 11.884$ & $135.73 \pm 7.847$ & $152.00 \pm 12.873$ & $<0.001$ & 0.229 & 0.001 & $<0.001$ \\
\hline & Insertion & $151.83 \pm 12.804$ & $140.87 \pm 7.646$ & $154.97 \pm 13.005$ & $<0.001$ & 0.001 & 0.873 & $<0.001$ \\
\hline & One minute & $151.27 \pm 12.795$ & $140.33 \pm 7.993$ & $155.50 \pm 13.054$ & $<0.001$ & 0.001 & 0.475 & $<0.001$ \\
\hline & Three minutes & $150.10 \pm 12.848$ & $139.40 \pm 7.972$ & $154.47 \pm 12.995$ & $<0.001$ & 0.002 & 0.436 & $<0.001$ \\
\hline & Five minutes & $149.03 \pm 12.931$ & $138.57 \pm 8.118$ & $153.20 \pm 13.158$ & $<0.001$ & 0.002 & 0.507 & $<0.001$ \\
\hline & Seven minutes & $149.50 \pm 12.905$ & $139.43 \pm 8.054$ & $154.33 \pm 13.682$ & $<0.001$ & 0.004 & 0.350 & $<0.001$ \\
\hline & Ten minutes & $150.70 \pm 13.233$ & $140.57 \pm 7.793$ & $155.60 \pm 13.768$ & $<0.001$ & 0.004 & 0.344 & $<0.001$ \\
\hline \multirow{4}{*}{ : } & PACU & $150.10 \pm 12.992$ & $140.10 \pm 7.774$ & $155.13 \pm 14.058$ & $<0.001$ & 0.005 & 0.317 & $<0.001$ \\
\hline & 15 minutes & $149.80 \pm 13.376$ & $140.00 \pm 8.204$ & $154.73 \pm 14.059$ & $<0.001$ & 0.007 & 0.360 & $<0.001$ \\
\hline & 30 minutes & $149.00 \pm 13.478$ & $139.70 \pm 8.408$ & $154.13 \pm 14.347$ & $<0.001$ & 0.014 & 0.334 & $<0.001$ \\
\hline & 60 minutes & $148.53 \pm 13.930$ & $139.23 \pm 8.169$ & $153.73 \pm 14.727$ & $<0.001$ & 0.016 & 0.342 & $<0.001$ \\
\hline
\end{tabular}

P1: NS group vs L group. P2: NS group vs LA group. P3: L group vs LA group.

Basal MAP was statistically insignificant when compared among the three groups $(p>0.05)$. Nevertheless, the LA group showed significantly higher MAP values during nebulization and induction ( $p=0.004$ and 0.001 respectively) in comparison to the other groups. On subsequent readings, no significant difference was noted between the three groups regarding the same parameter (table 3).

Table (3): Intra- and post-operative follow-up of MAP in the three groups.

\begin{tabular}{|c|c|c|c|c|c|c|c|c|}
\hline \multicolumn{2}{|c|}{ MAP (mmHg) } & NS group $(n=30)$ & $\mathrm{L}$ group $(\mathrm{n}=30)$ & LA group $(n=30)$ & $\mathbf{P}$ & P1 & P2 & P3 \\
\hline \multirow{9}{*}{ 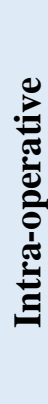 } & Basal & $63.53 \pm 5.513$ & $65.90 \pm 6.127$ & $63.83 \pm 7.679$ & 0.313 & 0.487 & 1 & 0.665 \\
\hline & Nebulizer & $63.60 \pm 5.587$ & $65.53 \pm 6.673$ & $69.67 \pm 8.384$ & 0.004 & 0.858 & 0.003 & 0.073 \\
\hline & Induction & $59.80 \pm 5.845$ & $61.70 \pm 7.349$ & $66.87 \pm 8.341$ & 0.001 & 0.939 & 0.001 & 0.021 \\
\hline & Insertion & $69.67 \pm 6.666$ & $68.33 \pm 7.893$ & $72.37 \pm 8.336$ & 0.122 & 1 & 0.528 & 0.134 \\
\hline & One minute & $70.03 \pm 6.851$ & $68.07 \pm 8.056$ & $72.60 \pm 8.295$ & 0.082 & 0.987 & 0.611 & 0.078 \\
\hline & Three minutes & $69.17 \pm 6.998$ & $67.10 \pm 7.761$ & $71.33 \pm 8.227$ & 0.108 & 0.900 & 0.832 & 0.107 \\
\hline & Five minutes & $68.37 \pm 6.835$ & $65.97 \pm 7.668$ & $70.27 \pm 8.292$ & 0.097 & 0.678 & 1 & 0.095 \\
\hline & Seven minutes & $69.37 \pm 6.851$ & $67.13 \pm 7.505$ & $71.30 \pm 8.575$ & 0.115 & 0.789 & 0.996 & 0.115 \\
\hline & Ten minutes & $70.13 \pm 6.606$ & $67.83 \pm 7.557$ & $72.37 \pm 8.503$ & 0.075 & 0.732 & 0.774 & 0.069 \\
\hline \multirow{4}{*}{$\stackrel{\dot{0}}{0}$} & PACU & $68.73 \pm 6.817$ & $67.63 \pm 7.416$ & $71.53 \pm 8.889$ & 0.139 & 1 & 0.497 & 0.164 \\
\hline & 15 minutes & $68.20 \pm 6.955$ & $67.10 \pm 7.640$ & $71.20 \pm 8.876$ & 0.119 & 1 & 0.429 & 0.140 \\
\hline & 30 minutes & $67.77 \pm 6.922$ & $66.60 \pm 7.837$ & $70.43 \pm 9.096$ & 0.170 & 1 & 0.601 & 0.201 \\
\hline & 60 minutes & $67.67 \pm 7.068$ & $66.07 \pm 7.570$ & $69.90 \pm 8.790$ & 0.170 & 1 & 0.819 & 0.185 \\
\hline
\end{tabular}

P1: NS group vs L group. P2: NS group vs LA group. P3: L group vs LA group.

$\mathrm{SPO}_{2}$ showed no significant differences among all groups (table 4). 
Table (4): Intra- and post-operative follow-up of $\mathrm{SPO}_{2}$ in the three groups.

\begin{tabular}{|c|c|c|c|c|c|c|c|c|}
\hline & $\mathrm{SPO}_{2}(\%)$ & NS group $(n=30)$ & $L$ group $(n=30)$ & LA group $(n=30)$ & $\mathbf{P}$ & P1 & $\mathbf{P 2}$ & P3 \\
\hline \multirow{9}{*}{ 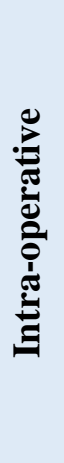 } & Basal & $96.43 \pm 1.25$ & $97.00 \pm 0.91$ & $97.97 \pm 0.85$ & 0.354 & 0.188 & 0.561 & 0.302 \\
\hline & Nebulizer & $95.70 \pm 1.39$ & $97.63 \pm 0.99$ & $97.00 \pm 0.87$ & 0.432 & 0.287 & 0.369 & 0.417 \\
\hline & Induction & $96.27 \pm 1.36$ & $97.40 \pm 1.16$ & $96.93 \pm 0.74$ & 0.448 & 0.311 & 0.117 & 0.121 \\
\hline & Insertion & $95.97 \pm 1.43$ & $97.30 \pm 1.18$ & $97.07 \pm 0.69$ & 0.441 & 0.255 & 0.295 & 0.326 \\
\hline & One minute & $96.03 \pm 1.42$ & $97.50 \pm 1.07$ & $96.97 \pm 0.89$ & 0.286 & 0.511 & 0.388 & 0.523 \\
\hline & Three minutes & $96.30 \pm 1.44$ & $97.60 \pm 1.10$ & $97.03 \pm 0.76$ & 0.128 & 0.367 & 0.184 & 0.415 \\
\hline & Five minutes & $95.63 \pm 1.22$ & $97.67 \pm 1.21$ & $97.00 \pm 0$ & 0.312 & 0.322 & 0.328 & 0.295 \\
\hline & Seven minutes & $96.53 \pm 1.19$ & $97.70 \pm 1.24$ & $96.77 \pm 0.82$ & 0.221 & 0.455 & 0.166 & 0.529 \\
\hline & Ten minutes & $95.97 \pm 1.49$ & $97.63 \pm 0.93$ & $97.20 \pm 0.66$ & 0.342 & 0.398 & 0.173 & 0.612 \\
\hline \multirow{4}{*}{$\stackrel{\dot{\Delta}}{0}$} & PACU & $96.33 \pm 1.42$ & $97.53 \pm 1.04$ & $97.10 \pm 0.76$ & 0.176 & 0.211 & 0.259 & 0.266 \\
\hline & 15 minutes & $95.97 \pm 1.45$ & $97.43 \pm 0.97$ & $96.90 \pm 0.80$ & 0.124 & 0.423 & 0.209 & 0.295 \\
\hline & 30 minutes & $96.17 \pm 1.66$ & $97.37 \pm 1.16$ & $97.13 \pm 0.82$ & 0.251 & 0.299 & 0.361 & 0.244 \\
\hline & 60 minutes & $96.40 \pm 1.35$ & $97.50 \pm 1.19$ & $96.96 \pm 0.85$ & 0.241 & 0.527 & 0.275 & 0.319 \\
\hline
\end{tabular}

P1: NS group vs L group. P2: NS group vs LA group. P3: L group vs LA group.

Propofol consumption showed a significant decline in two drug groups in comparison with the NS group ( $\mathrm{p}<0.001$ ). It had mean values of $61.83,42$, $45.67 \mathrm{mg}$ in the NS, L, and LA groups, respectively. The number of cases who developed intraoperative attacks of cough were higher in NS group when compared to L and LA groups respectively, meanwhile it showed no differences between both L and LA groups. In contrast the incidence of intraoperative desaturation and cough was incomparable among the studied groups. Post-operative RASS had median values of $1.5,2$, and 2 in the same groups, respectively,

Table (5): Intra-operative propofol requirements, cough, desaturation and post-operative recovery profile in the three groups.

\begin{tabular}{|c|c|c|c|c|c|c|c|}
\hline & $\begin{array}{l}\text { NS group } \\
(n=30)\end{array}$ & $\begin{array}{c}\text { L group } \\
(n=30)\end{array}$ & $\begin{array}{l}\text { LA group } \\
\quad(n=30)\end{array}$ & $\mathbf{P}$ & P1 & P2 & P3 \\
\hline $\begin{array}{l}\text { Propofol consumption } \\
\text { (mg) }\end{array}$ & $61.83 \pm 14.94$ & $42.00 \pm 8.47$ & $45.67 \pm 10.32$ & $<0.001$ & $<0.001$ & $<0.001$ & 0.669 \\
\hline $\begin{array}{l}\text { Intra-operative Cough } \\
\text { (number \& \% of cases) }\end{array}$ & $14(46.67 \%)$ & $9(30 \%)$ & $8(26.67 \%)$ & 0.028 & 0.011 & 0.261 & 0.374 \\
\hline $\begin{array}{l}\text { Average cough/case } \\
\text { (median \& range) }\end{array}$ & $3(1-4)$ & $2(1-3)$ & $2(1-2)$ & 0.106 & 0.667 & 0.212 & 0.245 \\
\hline $\begin{array}{c}\text { Intra-operative } \\
\text { desaturation } \\
\text { (number \& \% of cases) }\end{array}$ & $2(6.67 \%)$ & $1(3.33 \%)$ & $2(6.67 \%)$ & 0.630 & 1 & 1 & 0.964 \\
\hline $\begin{array}{l}\text { Post-operative RASS } \\
\text { (median \& range) }\end{array}$ & $1.50(1-2)$ & $2(1.75-2)$ & $2(2-2)$ & 0.014 & 0.018 & 0.096 & 0.732 \\
\hline $\begin{array}{l}\text { Post-operative Cough } \\
\text { (number \& \% of cases) }\end{array}$ & $13(43.3 \%)$ & $10(33.3 \%)$ & $3(10.0 \%)$ & 0.014 & $>0.05$ & $<0.05$ & $>0.05$ \\
\hline $\begin{array}{c}\text { Grade of Post-operative } \\
\text { Cough } \\
\text { (median \& range) }\end{array}$ & $7(6-8)$ & $5(3-6)$ & $3.5(2-5)$ & 0.001 & 0.002 & 0.001 & 0.003 \\
\hline $\begin{array}{l}\text { Post-operative stridor } \\
\text { (number \& \% of cases) }\end{array}$ & $3(10.0 \%)$ & $0(0.0 \%)$ & $1(3.33 \%)$ & 0.318 & $>0.05$ & $>0.05$ & $>0.05$ \\
\hline
\end{tabular}

with no significant difference between them $(\mathrm{p}=0.074)$. Regarding the post-operative period, the incidence of cough showed a significant decline in the LA group (10\% versus $43.3 \%$ and $33.3 \%$ in the NS and L groups, respectively). Similarly, cough severity was higher in NS group (sever cough) when compared to L (moderate cough) and LA (mild) group and when L (moderate) group were compared to LA (mild) group. However, the incidence of post-operative stridor was of no statistically significant values among the studied groups $(\mathrm{p}=0.318)$ as shown in table (5).

P1: NS group vs L group. P2: NS group vs LA group. P3: L group vs LA group. 


\section{DISCUSSION}

The removal of an aspirated foreign body is usually associated with prolonged manipulation of the upper respiratory tract, which leads to sympathetic overstimulation resulting in tachycardia, hypertension and cardiac arrhythmias ${ }^{20}$. Also, this manipulation is associated with airway oedema with subsequent narrowing ${ }^{21}$. This could be a risk of post-procedural respiratory adverse events ${ }^{7}$. Thus, it is essential to seek some maneuvers or interventions to decrease these complications.

Herein, we evaluated the effect of preoperative nebulization with lidocaine alone, compared to normal saline, and combined with epinephrine, regarding the incidence of post-operative respiratory complications.

Based on the previously mentioned results, it was noticed that there was no significant statistical differences between the three groups regarding all of the preprocedural parameters. This ensured the proper randomization technique, and this should also nullify any bias that might have skewed the results in favor of one group rather than the others.

In the current study, administration of nebulized lidocaine was associated with a significant decrease in heart rate compared to the NS and LA groups. This was evident from the time of intubation till the last follow up reading recorded. According to the existing literature, along with its local anesthetic action, the nebulized lidocaine could suppress the excitatory sensory $\mathrm{C}$ fibers present in the airway, leading to decreasing the stress response to laryngoscopy and bronchoscopy ${ }^{22-24}$.

In our study, administration of nebulized lidocaine was associated with a significant decrease in propofol consumption compared to the other two groups. In another study that evaluated the role of nebulized lidocaine in non-sedation bronchoscopy, nebulized lidocaine was associated with a significant increase in intraoperative sedation $(\mathrm{p}=0.04)$ compared to controls ${ }^{25}$.

Our findings showed that the administration of nebulized lidocaine was associated with a significant positive impact on intra-operative cough, but no significant effect on intra-operative desaturation, postoperative sedation and stridor ( $p>0.05$ ) compared to controls. The incidence and severity of postoperative cough was higher in NS group when compared to L and LA group and when L group was compared to LA group. In a previous similar study, the incidence of intra-operative cough did not show any significant differences between the lidocaine and saline groups ( $\mathrm{p}$ $=0.18$ ). Also, the incidence of post-operative complications, including hypoxia and excessive cough, was statistically comparable with the nebulized saline group $(\mathrm{p}=0.41)^{25}$. A previous Egyptian study reported that the nebulized lidocaine did not have any significant impact on the incidence of intraoperative cough or desaturation compared to controls who received only normal saline. However, nebulized fentanyl was associated with better hemodynamic response and decreased incidence of cough. Authors attributed these beneficial effects to the opioid characteristics of fentanyl. However, patients receiving fentanyl needed more time to full wakefulness ${ }^{15}$. On the other hand, Palva et al. ${ }^{26}$ revealed the efficacy and improved patient acceptability in participants undergoing bronchoscopy with nebulized lignocaine. Following that, further studies have been conducted to assess the role of nebulized lignocaine in patients undergoing bronchoscopy.

When it comes to lidocaine adrenaline combination in the current study, its administration via nebulization was associated with significantly higher heart rates compared to the two groups. Also, MAP showed a significant rise during nebulization and anaesthetic induction. However, the post-hoc analysis revealed no comparable findings with the control group $(p>0.05)$ in most readings. In line with our findings, Numa et al. ${ }^{27}$ reported a significant increase in heart rates after receiving nebulized epinephrine in the included 15 pediatric patients diagnosed with bronchiolitis. It was increased from $145 \mathrm{bpm}$ (range, 94 - 177) before epinephrine, up to $164 \mathrm{bpm}$ (range, 118 $203)$ after it $(\mathrm{p}=0.0008)$. The same authors also reported a subtle increase in MAP after epinephrine nebulization in their study $(p=0.055)$. It increased from $53 \mathrm{mmHg}$ before the nebulizer up to $59 \mathrm{mmHg}$ after it. The previous study reported a much stronger impact of epinephrine on the studies parameters, and we could attribute the weak effect in our study to its combination with lidocaine, which helped to attenuate the stress response associated with adrenaline injection. This could also explain the decreased propofol consumption in the LA group compared to controls.

In our study, although the LA group showed comparable results regarding intra-operative adverse events, there was a significant decrease in the incidence of post-operative cough in this group $(\mathrm{p}=0.014)$. Adrenaline has a stimulant action for both alpha and beta receptors, which have a beneficial impact on both bronchial blood vessels and muscles. This, in turn led to the relief of airway obstruction resulting from bronchoscope manipulation ${ }^{28}$. Other studies also demonstrated the beneficial impact of epinephrine nebulization in children with bronchiolitis. It was associated with improved respiratory functions and lung mechanics ${ }^{27,28}$. This could explain our finding.

Although our study revealed no significant difference between the three groups regarding postprocedural stridor, its incidence was higher in the NS group. In the same context, a recent report even stated that nebulized epinephrine (1:1000) could be used if patients developed stridor following bronchoscopy ${ }^{29}$.

Our study had some limitations where it is a single-center study that included a relatively small sample size. More studies, including more cases with different nebulized drugs, should be performed to 
define the best-nebulized regimen that should be commenced for such cases before bronchoscopy.

\section{CONCLUSION}

Based on our findings, nebulized lidocaine/adrenaline combination can provide proper intraoperative sedation and upper airway conditions together with reduction in post-operative unwanted respiratory outcomes, with minor hemodynamic changes during pediatric rigid bronchoscopy procedures.

\section{Declaration of interest}

The authors have no conflict of interest.

Financial support and sponsorship: Nil.

\section{REFERENCES}

1. Fidkowski C, Zheng H, Firth P (2010): The anesthetic considerations of tracheobronchial foreign bodies in children: a literature review of 12,979 cases. Anesth Analg., 111 (4): 1016-25.

2. Hitter A, Hullo E, Durand C, Righini C (2011): Diagnostic value of various investigations in children with suspected foreign body aspiration: review. Eur Ann Otorhinolaryngol Head Neck Dis., 128 (5): 248-52.

3. Clerf L (1975): Historical aspects of foreign bodies in the air and food passages. South Med J., 68 (11): 1449-54.

4. Na'ara S, Vainer I, Amit M, Gordin A (2020): Foreign Body Aspiration in Infants and Older Children: A Comparative Study. Ear, nose, \& throat journal, 99 (1): 4751.

5. Golan-Tripto I, Mezan D, Tsaregorodtsev S, StilerTimor L, Dizitzer Y, Goldbart A et al. (2021): From rigid to flexible bronchoscopy: a tertiary center experience in removal of inhaled foreign bodies in children. Eur J Pediatr., 180 (5): 1443-50.

6. Gorden J (2013): Rigid Bronchoscopy. In: Ernst A, Herth FJF, editors. Principles and Practice of Interventional Pulmonology. New York, NY: Springer New York, Pp: 28595.

7. Batra H, Yarmus L (2018): Indications and complications of rigid bronchoscopy. Expert Rev Respir Med., 12 (6): 50920.

8. Faro A, Wood R, Schechter M, Leong A, Wittkugel E, Abode K et al. (2015): Official American Thoracic Society technical standards: flexible airway endoscopy in children. Am J Respir Crit Care Med., 191 (9): 1066-80.

9. Rovin J, Rodgers B (2000): Pediatric foreign body aspiration. Pediatr Rev., 21 (3): 86-90.

10. Sersar S, Rizk W, Bilal M, El Diasty M, Eltantawy T, Abdelhakam B et al. (2006): Inhaled foreign bodies: presentation, management and value of history and plain chest radiography in delayed presentation. Otolaryngol Head Neck Surg., 134 (1): 92-9.

11. Zaytoun G, Rouadi P, Baki D (2000): Endoscopic management of foreign bodies in the tracheobronchial tree: predictive factors for complications. Otolaryngol Head Neck Surg., 123 (3): 311-6.

12. Aydoğan L, Tuncer U, Soylu L, Kiroğlu M, Ozsahinoglu C (2006): Rigid bronchoscopy for the suspicion of foreign body in the airway. Int $\mathbf{J}$ Pediatr Otorhinolaryngol., 70 (5): 823-8.

13. Ganie F, Wani M, Ahangar A, Lone G, Singh S, Lone H et al. (2014):The Efficacy of Rigid Bronchoscopy for Foreign Body Aspiration. Bull Emerg Trauma., 2 (1): 52-4
14. Shaffer T, Wolfson M, Panitch H (2004): Airway structure, function and development in health and disease. Paediatr Anaesth., 14 (1): 3-14.

15. Moustafa M (2013): Nebulized lidocaine alone or combined with fentanyl as a premedication to general anesthesia in spontaneously breathing pediatric patients undergoing rigid bronchoscopy. Paediatr Anaesth., 23 (5): 429-34.

16. Klassen $T$ (1997): Recent advances in the treatment of bronchiolitis and laryngitis. Pediatr Clin North Am., 44 (1): 249-61.

17. Brown J (2002): The management of croup. Br Med Bull., 61: 189-202.

18. Abul-Ainine A, Luyt D (2002) Short term effects of adrenaline in bronchiolitis: a randomized controlled trial. Arch Dis Child., 86 (4): 276-9.

19. Kerson A, DeMaria R, Mauer E, Joyce C, Gerber L, Greenwald B et al. (2016): Validity of the Richmond Agitation-Sedation Scale (RASS) in critically ill children. J Intensive Care, 4: 65.

20. Matot I, Sichel J, Yofe V, Gozal Y (2000): The effect of clonidine premedication on hemodynamic responses to microlaryngoscopy and rigid bronchoscopy. Anesth Analg., 91 (4): 828-33.

21. Chaddha U, Murgu S (2021): Complications of rigid bronchoscopy. Respirology, 26 (1): 14-8.

22. Bidwai A, Bidwai V, Rogers C, Stanley T (1797): Bloodpressure and pulse-rate responses to endotracheal extubation with and without prior injection of lidocaine. Anesthesiology, 51 (2): 171-3.

23. Kumar A, Seth A, Prakash S, Deganwa M, Gogia A (2016): Attenuation of the hemodynamic response to laryngoscopy and tracheal intubation with fentanyl, lignocaine nebulization, and a combination of both: A randomized controlled trial. Anesth Essays Res., 10 (3): 661-6.

24. Gulabani M, Gurha P, Dass P, Kulshreshtha N (2015): Comparative analysis of efficacy of lignocaine $1.5 \mathrm{mg} / \mathrm{kg}$ and two different doses of dexmedetomidine $(0.5 \mu \mathrm{g} / \mathrm{kg}$ and $1 \mu \mathrm{g} / \mathrm{kg}$ ) in attenuating the hemodynamic pressure response to laryngoscopy and intubation. Anesth Essays Res., 9 (1): 5-14.

25. Madan K, Biswal S, Tiwari P, Mittal S, Hadda V, Mohan A et al. (2019): Nebulized lignocaine for topical anaesthesia in no-sedation bronchoscopy (NEBULA): A randomized, double blind, placebo-controlled trial. Lung India, 36 (4): 288-94.

26. Palva T, Jokinen K, Saloheimo M, Karvonen P (1975): Ultrasonic nebulizer in local anesthesia for bronchoscopy. ORL J Otorhinolaryngol Relat Spec., 37 (5): 306-11.

27. Numa A, Williams G, Dakin C (2001): The effect of nebulized epinephrine on respiratory mechanics and gas exchange in bronchiolitis. Am J Respir Crit Care Med., 164 (1): 86-91.

28. Lødrup Carlsen K, Carlsen K (2000): Inhaled nebulized adrenaline improves lung function in infants with acute bronchiolitis. Respir Med., 94 (7): 709-14.

29. Johnson M, Sims C (2020): bronchoscopy for foreign bodyBronchoscopy and Removal of Foreign Bodies from the Trachea. In: Sims C, Weber D, Johnson C, editors. A Guide to Pediatric Anesthesia. Cham: Springer International Publishing, Pp: 351-63. 